\title{
Real time ultrasonography of the gastric antrum
}

\author{
R Ricci, I Bontempo, E Corazziari, A La Bella, A Torsoli
}

\footnotetext{
Cattedra di

Gastroenterologia I,

Clinica Medica II,

Università La Sapienza,

Roma, Italy

R Ricci

I Bontempo

E Corazziari

A La Bella

A Torsoli

Correspondence to:

Dr E Corazziari, Cattedra di

Gr E Corazziari, Cattedra di

Medica II, Università di Roma

Medica II, Università di
La Sapienza, Viale del

Policlinico, 00161 ROMA,

Policli
Italy.

Accepted for publication

13 July 1992
}

\begin{abstract}
This study aimed to evaluate whether serial ultrasound measurements of antral volumes are reproducible and, if so, whether they are representative of the entire gastric contents and reliably measure gastric emptying. The antral volume was measured in man after oral and intragastric administration of known amounts of $5 \%$ glucose solutions, and on two separate occasions in the same subjects, after the ingestion of a standardised solid-liquid meal $(1050 \mathrm{kcal})$. Antral volume measurements were performed in both supine and upright positions; inter- and intraobserver measurement errors were also assessed. After ingestion or intragastric administration of equal amounts of liquid, antral volumes determined by ultrasound showed a wide intersubject variability. In each individual subject, however, a linear trend was found between the antral volume and amount of ingested or administered liquid. Intrasubject variability of antral volume measurements was at its minimum in fasting conditions and $\mathbf{3 0 0}$ minutes after ingestion of the solid-liquid meal. Intra- and interobserver variations and whether the patient was erect or supine did not affect measurements of antral volumes. These results support the use of real time ultrasound in determining the gastric emptying time. Results at fasting and 300 minutes after a solid-liquid meal are stable end points of measurement.

(Gut 1993; 34: 173-176)
\end{abstract}

Real time ultrasonography has recently been used to assess gastric motility ${ }^{1-1}$ and gastric emptying. ${ }^{2-4}$ Measurements of the volume of the entire stomach were first suggested as a means of evaluating gastric emptying. This approach, however, is greatly limited by technical difficulties and potential inaccuracies. The technique is tedious, and time consuming; a microcomputer is desirable to facilitate measurement, the technique can fail when intragastric gas is present, and it can only be used after liquid meals. ${ }^{5}$ In an attempt to overcome these limitations it has been suggested that gastric emptying should be derived from changes in antral volume. ${ }^{6}$ Ultrasound measurements of the antral volume can be undertaken in any subject, irrespective of gas contents in the fundus, and can also be used after any type of meal. ${ }^{6}$ It remains to be established whether the antral volume is representative of the entire gastric contents and whether its serial quantitation can reliably express modalities of gastric emptying.

In this study we tried to determine whether ultrasound measurement of the gastric antrum volume is representative of the volume of the ingested material and of the entire gastric contents and whether it is reproducible and affected by body position.

\section{Subjects and methods}

\section{SUBJECTS}

Ten healthy subjects (five men and five women: mean age 28 years; range $24-39$ years) and five female patients with dyspepsia (mean age 41 years; range 35-47 years) were investigated. Healthy subjects were asymptomatic and had no evidence of gastrointestinal diseases or other diseases affecting gastric motility. In these subjects abdominal ultrasound examination also proved negative. Dyspeptic patients reported postprandial epigastric discomfort and feelings of postprandial food retention in the stomach. Upper gastrointestinal radiology, endoscopy, and abdominal ultrasound, proved negative in this group. The study was approved by the Ethical Committee of Clinica Medica II, Università La Sapienza, Roma and all subjects gave their written informed consent.

\section{STUDY PROTOCOL}

The antrum volume (expressed in $\mathrm{ml}$ ) was obtained by the following formula as discussed in detail by Bolondi et $a l^{6}$ and based on the presumption that every antral section is elliptical and that the variations of the axis are linear:

$0.065 \mathrm{xh} \times(2 \mathrm{ab}+2 \mathrm{ef}+4 \mathrm{~cd}+\mathrm{cb}+\mathrm{ad}+\mathrm{ed}+\mathrm{cf})$

The symbols are explained in the legend to Figure 1.

The orientation of the antrum may differ in any individual subject; consequently various degrees of slope were used by the ultrasound probe to obtain the correct scans. The measurements were taken from the inner side of the antrum wall. To perform a correct scan at the level of the angulus the probe is swept from right to left along the antrum while maintained orthogonal to its longitudinal axis.

The antral section is considered to be located at the angulus just before image disappearance.

All subjects were investigated after an overnight fast of at least eight hours. Smoking was prohibited during the test.

The following tests were performed:

(1) Antral volume was measured in 10 healthy subjects after oral ingestion of successive amounts of $100 \mathrm{ml} \mathrm{5 \%}$ glucose solution up to a maximum of $600 \mathrm{ml}$. Each ingestion of the $100 \mathrm{ml}$ solution was immediately followed by ultrasound measurement. The time to perform the entire sequence of measurements did not exceed 15 minutes in any individual and was performed in the upright position.

(2) In five dyspeptic patients a nasogastric catheter (Kaslow, Levin type; size $5.1 \mathrm{~mm}$, 


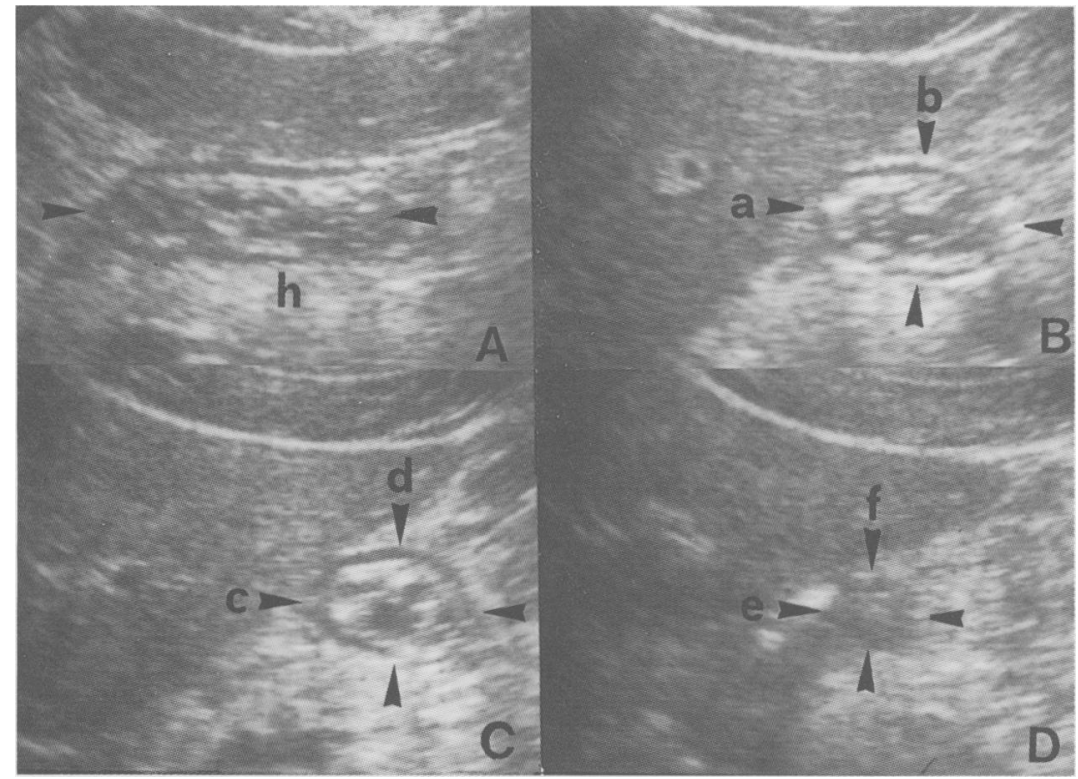

Figure 1: Ultrasound scan of the gastric antrum during fasting. In $(A)$ the longitudinal section obtained by means of an epigastric transverse scan measures the antral length $(h)$. These axial sections are shown in $B, C$, and $D ;(B)$ the angulus level section; a being the craniocaudal and $\mathrm{b}$ the anteroposterior diameters; $(C)$ intermediate level of the antrum section; $\mathrm{c}$ being the craniocaudal and $\mathrm{d}$ the anteroposterior diameters; $(D)$ prepyloric level section; $\mathrm{e}$ being the craniocaudal and $\mathrm{f}$ the anteroposterior diameters.

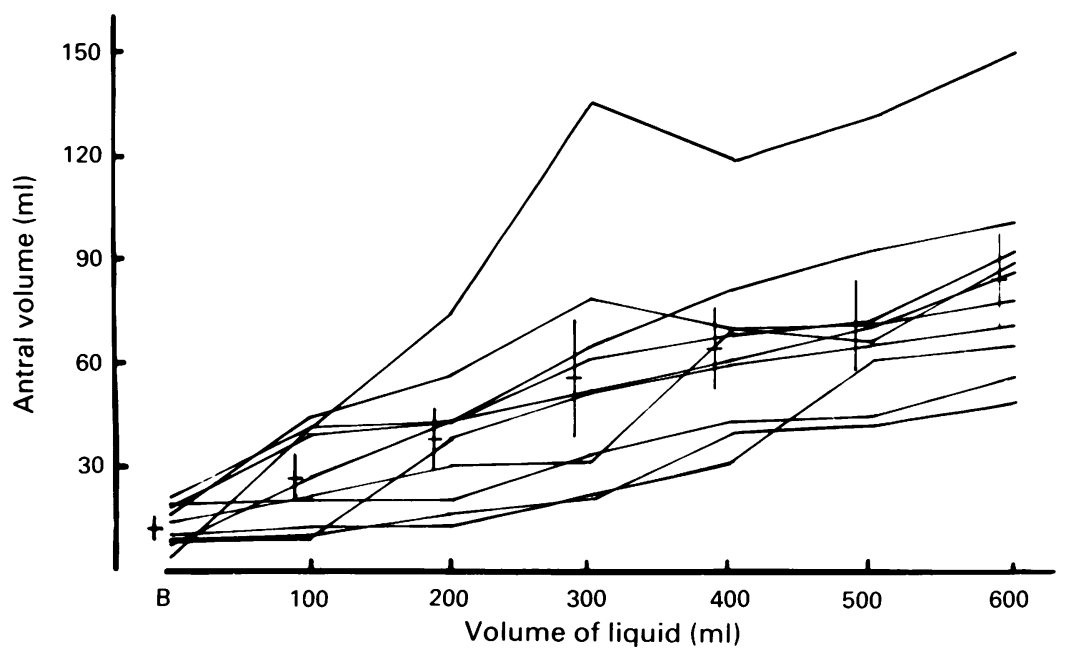

Figure 2: Ultrasound antral volumes of each individual subject in basal conditions $(B)$ and after ingestion of known increasing amounts of liquid. Mean $(S D)$ are indicated by horizontal and vertical bars.

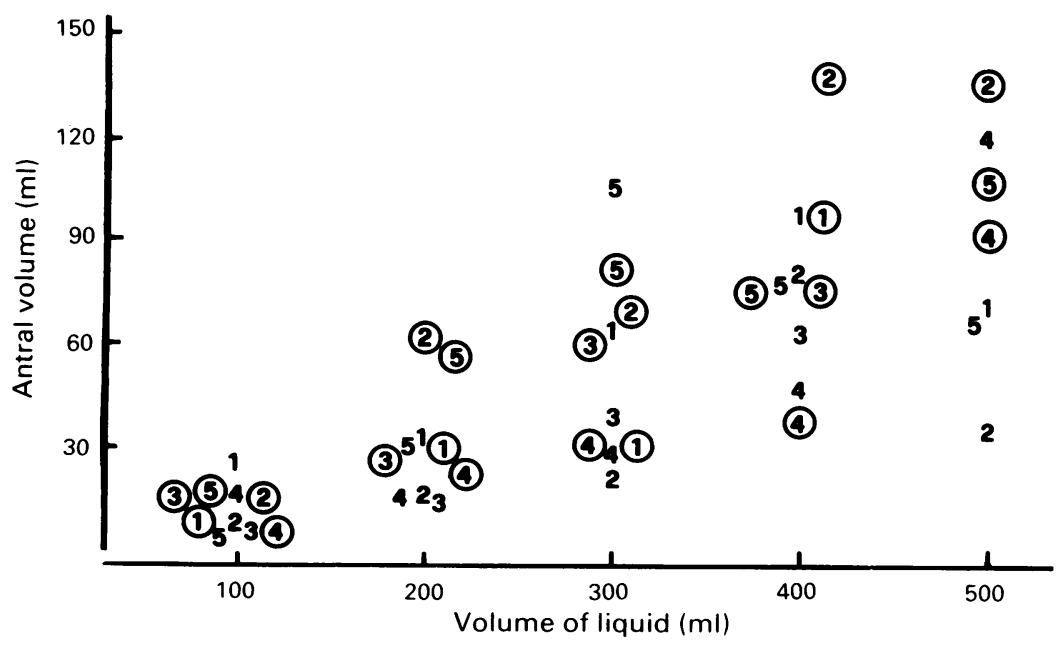

Figure 3: Ultrasound volume of each individual subject after the infusion of known amounts of liquid through a nasogastric catheter into the stomach. At $500 \mathrm{ml}$ three measurements were not evaluated. Each circled and uncircled number identifies a series of solution administration in an individual patient. length $120 \mathrm{~cm}$ ) was passed from one nostril into the stomach and was positioned under fluoroscopic control with the aspirating holes in the lowest part of the stomach. One hundred, 200, 300,400 , and $500 \mathrm{ml}$ of $5 \%$ glucose solution were introduced into the stomach after aspiration of the gastric contents. Each time the solution was administered complete aspiration of the gastric contents followed. Ultrasound measurement was performed immediately after each intragastric administration and after each aspiration with the patient in upright position. During this study the operator was unaware of the amount of liquid administered. In each subject two series of solutions ( 100 to $500 \mathrm{ml}$ ) were given, and in each series solutions were administered in random order.

(3) On two different days at two week intervals antral volume was measured in 10 healthy subjects. It was measured under fasting conditions and at $0,60,120,180,240$, and 300 minutes after the ingestion of a solid-liquid meal. The meal consisted of bread (140 g), Emmenthal cheese $(70 \mathrm{~g})$, ham $(80 \mathrm{~g})$, Albios Giuliani crackers $(18 \mathrm{~g})$, and plain water $(250 \mathrm{ml})$ giving carbohydrate $(100 \mathrm{~g})$; protein $(50 \mathrm{~g})$, lipid $(50 \mathrm{~g})$, and fibre $(4.0 \mathrm{~g}),(1050 \mathrm{kcal})$. At each ultrasound observation measurements were performed in both the supine and upright positions.

(4) To evaluate the interobserver error, two independent sonographers performed antral volume measurements in a rapid time sequence during additional studies in six healthy subjects before and after the ingestion of the standard solid-liquid meal. In addition, three independent sonographers performed five antral volume measurements in a rapid time sequence during one of the tests in a control subject.

\section{STATISTICAL ANALYSIS}

Intragroup comparisons were assessed with the Wilcoxon test. Observer variability was assessed with the analysis of variance and the Wilcoxon test.

\section{Results}

Ultrasound antral volumes after ingestion of known, increasing amounts of liquid and after intragastric administration of known amounts of liquid and after fasting, are reported in Figures 2 and 3 . Ultrasound antral volumes differed widely from subject to subject after meal ingestion and also after administration of equal amounts of liquid. Volumes showed no relation to age, sex, body weight, or height. Overall the antral volumes increase as the amount of ingested or administered liquid increases. In each individual subject a linear trend was found between antral volumes and the amount of liquid, except for a small deviation from linearity in three of the 10 tests in which the solutions were administered directly into the stomach.

The ultrasound scans of the posterior wall of the antrum after a liquid-solid meal were less clearly defined than those taken after a liquid meal. As digestion of the gastric contents progressed, the posterior wall of the antrum became more evident. Figure 4 shows a time sequence of 


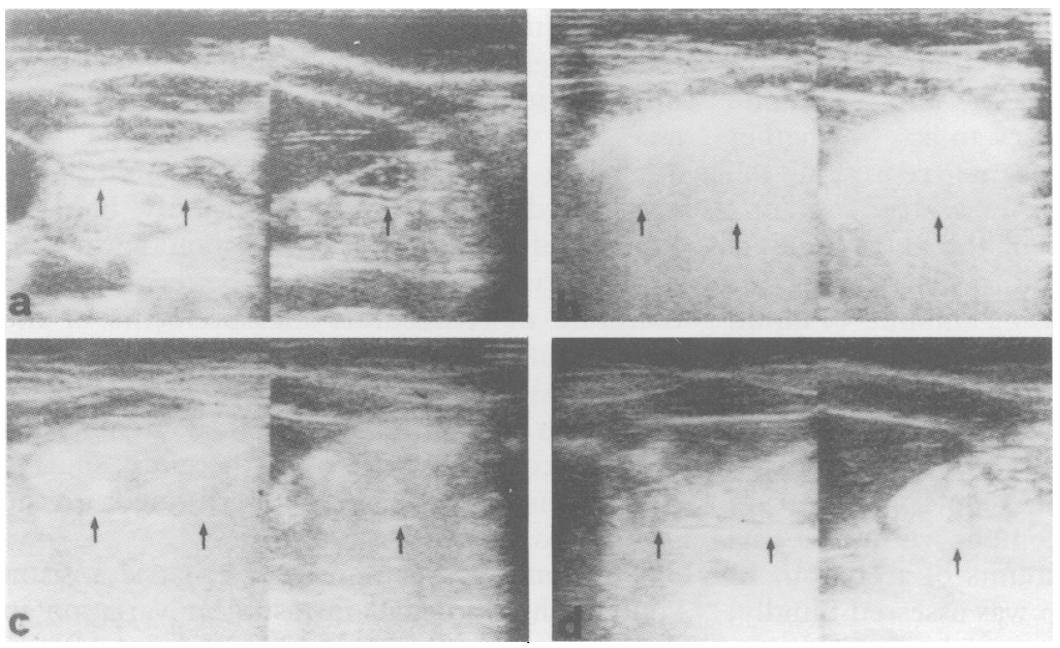

Figure 4: Ultrasound scan of the gastric antrum in fasting condition $(a)$ : immediately after $(b)$, at 180 minutes (c), and 300 minutes (d) after the standard meal. At each time, on the left: longitudinal scan of the gastric antrum; on the right: transverse scan of the gastric antrum at the level of the angulus. The arrows indicate the posterior wall of the gastric antrum which is well delimited in fasting conditions (a) and 180-300 minutes after meal ingestion (c, d).

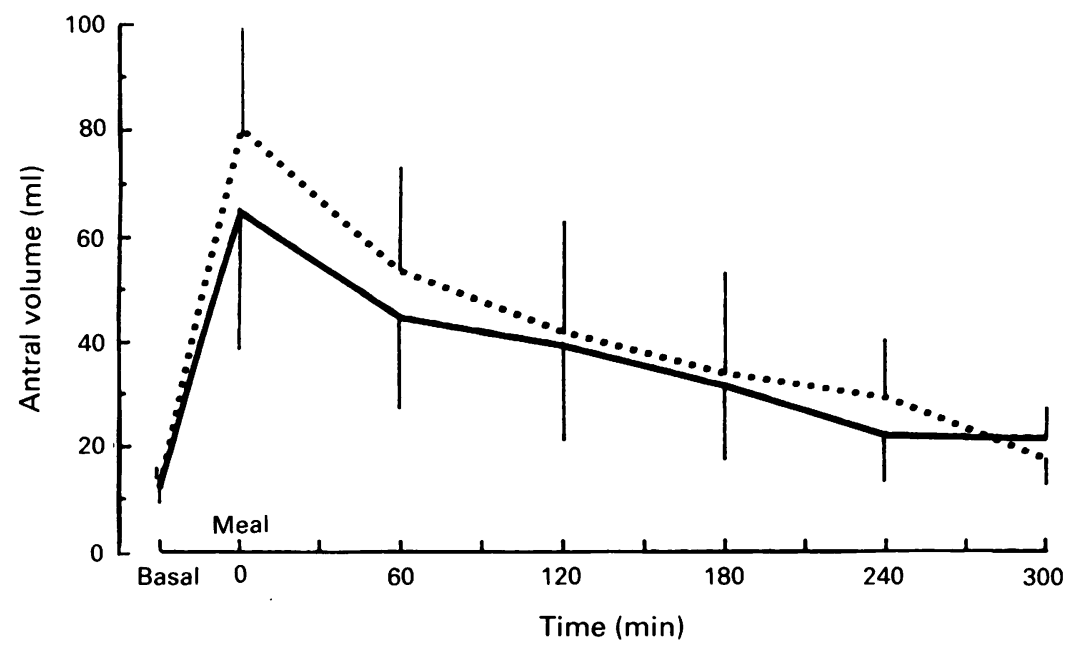

Figure 5: Ultrasound volume (mean (SD)) measured in the supine position before (basal) and after the standard meal in the duplicate studies (dotted line: second study).

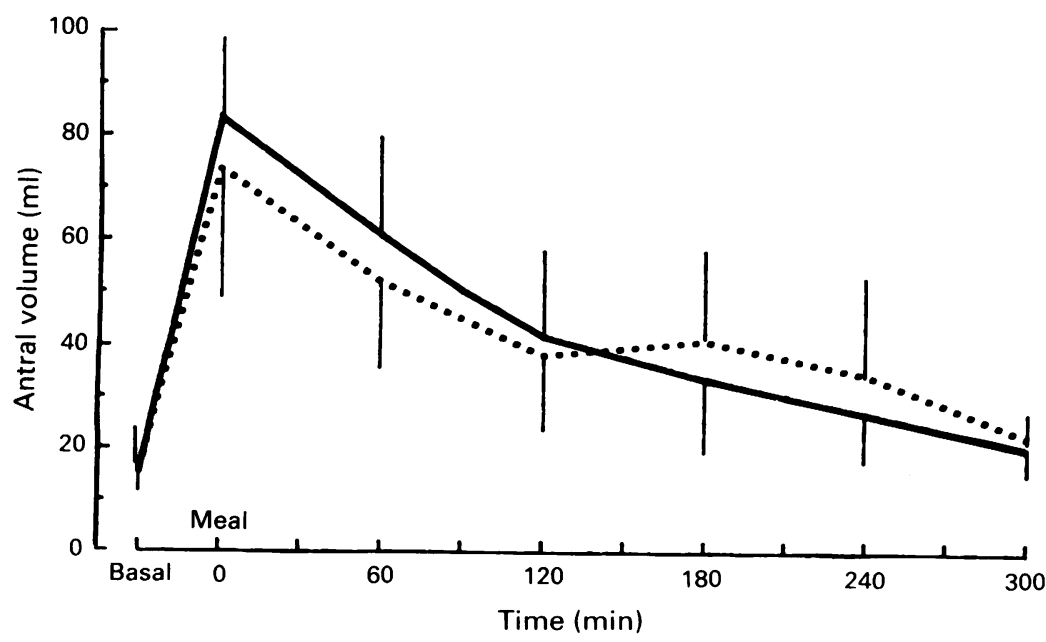

Figure 6: Ultrasound volume (mean (SD)) measured in the upright position before (basal) and after the standard meal in the duplicate studies (dotted line: second study). gastric antrum scans taken before and after meal ingestion. The mean ultrasound antral volumes of healthy fasted subjects were 12 and $15 \mathrm{ml}$ respectively in supine and upright positions. The antral volume reached its highest value after meal ingestion ( 30 minutes after the beginning of the meal) and then fell progressively in a linear manner. At 300 minutes after the meal, the antral volume showed a mean (SD) reduction of 85 (12)\% (range 60-100\%) compared with the postprandial values.

The mean (SD) antral volumes in both the supine and upright positions, and before and after standard liquid-solid meal, are given in Figures 5 and 6 for the first and second studies respectively. The volume measurements taken in the upright position were often larger than those taken in the supine position but no statistical differences could be found.

Variations in antral volume measurements performed in the duplicate studies (expressed as delta $\mathrm{ml}$ ), with reference to body position are reported in Table I. There was no significant difference between mean values of the two studies at any time interval, but for individual cases the greatest variation between the two studies occurred immediately after meal ingestion and continued for a further 240 minutes.

Antral volume measurements, performed in a rapid time sequence by two independent sonographers, are reported in Table II and did not show any statistical difference. Moreover the measurements of any single study showed a highly significant and direct relation between the two observers ( $\mathrm{r}$ value of any single study: $0 \cdot 71$, $0.90,0.96,0.72,0.90,0.84)$. In addition the rapid time sequence measurements in the same subject obtained by three independent sonographers did not show any statistical difference $(\mathrm{F}=0.03)$.

\section{Discussion}

Ultrasound can be used to measure antral volume, and serial quantitation of the antral volume after a meal has been proposed as a

TABLE I Delta variations mean (SD), (range) in antral volume ( $\mathrm{ml}$ ) between measurements performed in the duplicate studies in the supine and upright positions

\begin{tabular}{lcc}
\hline Time & Supine position & Upright position \\
\hline Baseline & $3 \cdot 3(1 \cdot 9)(0 \cdot 6-6 \cdot 2)$ & $5 \cdot 5(4 \cdot 1)(0 \cdot 6-13 \cdot 3)$ \\
After meal & $24 \cdot 3(15 \cdot 7)(0 \cdot 5-45 \cdot 7)$ & $27 \cdot 8(21 \cdot 5)(1 \cdot 9-72 \cdot 3)$ \\
At 60 min & $17 \cdot 4(10 \cdot 7)(1 \cdot 4-36 \cdot 3)$ & $20 \cdot 7(19 \cdot 1)(2 \cdot 8-85 \cdot 0)$ \\
At 120 min & $17 \cdot 0(13 \cdot 2)(1 \cdot 9-41 \cdot 1)$ & $15 \cdot 2(16 \cdot 4)(0 \cdot 5-48 \cdot 3)$ \\
At 180 min & $11 \cdot 7(7 \cdot 9)(3 \cdot 3-27 \cdot 1)$ & $11 \cdot 2(10 \cdot 4)(2 \cdot 4-35 \cdot 2)$ \\
At 240 min & $8 \cdot 5(6 \cdot 9)(1 \cdot 7-23 \cdot 4)$ & $13 \cdot 0(8 \cdot 1)(2 \cdot 0-27 \cdot 8)$ \\
At 300 min & $6 \cdot 4(6 \cdot 1)(0 \cdot 4-16 \cdot 8)$ & $5 \cdot 0(3 \cdot 9)(0 \cdot 6-10 \cdot 7)$ \\
\hline
\end{tabular}

TABLE II Ultrasound measurements of antral volume $(\mathrm{ml}$; mean $(S D)$ ) performed independently by two sonographers

\begin{tabular}{lll}
\hline Time & First & Second \\
\hline Baseline & $11 \cdot 5(7 \cdot 5)$ & $13 \cdot 3(6 \cdot 2)$ \\
After meal & $95 \cdot 3(29 \cdot 4)$ & $82 \cdot 9(35 \cdot 9)$ \\
At $60 \mathrm{~min}$ & $57 \cdot 1(12 \cdot 8)$ & $56 \cdot 8(13 \cdot 3)$ \\
At $120 \mathrm{~min}$ & $45 \cdot 2(15 \cdot 1)$ & $48 \cdot 9(10 \cdot 8)$ \\
At $180 \mathrm{~min}$ & $45 \cdot 8(16 \cdot 8)$ & $47 \cdot 9(14 \cdot 4)$ \\
At $240 \mathrm{~min}$ & $42 \cdot 5(19 \cdot 2)$ & $47 \cdot 1(16 \cdot 0)$ \\
At $300 \mathrm{~min}$ & $21 \cdot 9(9 \cdot 1)$ & $20 \cdot 5(3 \cdot 2)$ \\
\hline
\end{tabular}


means of determining the gastric emptying time. ${ }^{6}$ To express gastric emptying time in terms of sequential measurement of the antral volume, however, it is first necessary to assess whether the ultrasound measurements represent the ingested meal and the entire gastric contents. Ultrasound measurement of the antral volume was therefore performed immediately after the ingestion of known increasing amounts of liquid and, in different subjects, immediately after the intragastric administration of different amounts of liquid to an empty stomach. This second set of experiments could assess the relationship between ultrasound measurements of the antrum and the entire gastric content without being affected by unknown amounts of secretion. In addition, this relationship was assessed blindly, irrespective of the sequence of administration.

After both oral ingestion and intragastric administration of equal amounts of liquid, the antral volume showed wide intersubject variation that did not seem to be related to sex, age, or body size. For each individual subject, however, a linear trend was found between the antral volume and the ingested or administered liquid, thus indicating that the sequential measurements of the antral volumes after ingestion of a meal may express the modality of variation of the gastric contents.

This study then assessed the intrasubject and time related variability of ultrasound antral measurements comparing data derived from separate studies performed in the same experimental conditions, in the same subject, and after ingestion of an identical standard meal.

The study shows that ultrasound measurement of antral volume shows a wide individual difference both in basal fasting conditions and after meal ingestion. At 300 minutes, after meal ingestion, however, all normal subjects showed at least a $60 \%$ reduction in the postprandial antral volume. Intrasubject variability was at its maximum immediately after meal ingestion and then to a lesser degree, up to 180 minutes after the meal. Similar temporal intrasubject variation in gastric emptying in healthy subjects has been previously described by scintigraphic methods ${ }^{7}$ and may simply reflect the day to day fluctuations in gastric emptying rate. Part of this variation, however, may derive from the difficulty in accurately defining the posterior wall of the antrum until various components of the meal become homogenised. The extent and duration of this type of inaccuracy would, therefore, depend on the specific components of the meal and on how long it takes for them to become homogenised. A more liquid or semisoft meal probably allows better and earlier mixing of food within the stomach and better visualisation of the posterior gastric wall. Under the present experimental conditions, in which the meal (an average lunch) was composed of solid particles with a relatively low amount of liquid, the inaccuracy in identifying the posterior wall of the antrum was confined, on average, to the 180 minutes after a meal.

The variation in measurements because of intraobserver and interobserver variation seems minimal since measurements of the same observer in duplicate studies and of two or three independent observers in the same study did not differ significantly. Finally the present study assessed whether antral measurements vary according to body position. Ultrasound antral volumes did not differ significantly between supine and upright positions. It should be noted, however, that in some subjects better scans, and therefore more accurate measurements of the gastric antrum, were obtained in the upright position, since this allowed for a more satisfactory separation of gas from liquid/solid contents. This practical consideration would, therefore, suggest that ultrasound should be performed in the upright position.

Ultrasound measurements of gastric antrum volume show a small intrasubject variation in fasting conditions and at 300 minutes after a standard meal. Our data seem to support the use of real time ultrasound in the measurement of gastric antrum volume and, thus the possibility of evaluating the emptying modalities by means of serial quantitation of the antral volume, even following the ingestion of a solid meal after the posterior wall of the gastric antrum becomes visible. According to the data presented here, real time ultrasound enables quantitative assessment of gastric emptying of a standard solidliquid meal when serial measurements are performed late after ingestion of a meal using the same components as in this study. A measurement at a predefined time after eating a standard meal could be used as an end point - for example 300 minutes after the ingestion of the present standard meal one would expect the stomach to be at least $60 \%$ empty.

Compared with ultrasound measurement of the entire stomach, assessing of antral volume guarantees visualisation of the antrum, it can be used with any kind of meal (both solid and/or liquid), and it needs no computer assistance. Therefore, ultrasound antral volume measurement seems to be a simple, non-invasive, inexpensive and undemanding method, that can offer reliable indications on the modality of gastric emptying.

The authors express their gratitude to Dr Flavia Mascioli for statistical help.

1 Holt S, McDicken WN, Anderson T, Stewart IC, Heading RC. Dynamic imaging of the stomach by real time ultrasound - a method for the study of gastric motility. Gut 1980; 21: $597-$ 601 .

2 Marzio L, Neri M, Di Gianmarco AM, Cuccurullo F. Evaluation of the gastric emptying and motility by means of real time ultrasonography. In: Labò S, Bolondi L, Rizzatto S, eds. ultrasonography. In: Labò S, Bolondi L, Rizzatto S, eds. Clinical
$167-74$.

3 King PM, Adam RD, Pryde A, McDicken WN, Heading RC Relationship of human antroduodenal motility and tran pyloric fluid movement: non-invasive observations with real time ultrasound. Gut 1984; 25: 1384-91.

4 Adam RD, McDicken WN, Anderson T, Heading RC. Antral contractions and fluid movement in the human stomach. Observations with real time ultrasound. In: Labò G, Bortolotti M, eds. Gastrointestinal motility. Proceedings of the First European Symposium on Gastrointestinal Motility. Verona: Cortina International, 1983: 255-6.

5 Holt S, Cervantes J, Wilkinson AA, Wallace JHK. Measurement of gastric emptying rate in humans by real-time ment of gastric emptying rate in humans
ultrasound. Gastroenterology 1986; $90: 918-23$.

6 Bolondi L, Bortolotti M, Santi V, Calletti T, Gaiani S, Labò G. Measurement of gastric emptying time by real-time ultra

7 Brophy CM, Moore JG, Christian PE, Egger MJ, Taylor AT Variability of gastric emptying measurements in man employing standardized radiolabeled meals. Dig Dis Sci 1986; 31: 799-806. 